\title{
Carriers of Sargassum and mechanism for coastal inundation in the Caribbean Sea
}

\author{
F. Andrade-Canto \\ Departamento de Observación y \\ Estudio de la Tierra, la Atmósfera y \\ el Océano \\ El Colegio de la Frontera Sur \\ Chetumal, Quintana Roo, Mexico \\ fernando.andrade@ecosur.mx \\ G.J. Goni \\ Atlantic Oceanographic and \\ Meteorological Laboratory \\ National Oceanic \& Atmospheric \\ Administration \\ Miami, Florida, USA \\ gustavo.goni@noaa.gov \\ M.J. Olascoaga \\ Department of Ocean Sciences \\ Rosenstiel School of Marine \& \\ Atmospheric Science \\ University of Miami \\ Miami, Florida, USA \\ jolascoaga@miami.edu
}

\author{
F.J. Beron-Vera \\ Department of Atmospheric Sciences \\ Rosenstiel School of Marine \& \\ Atmospheric Science \\ University of Miami \\ Miami, Florida, USA \\ fberon@miami.edu \\ D. Karrasch \\ Technische Universität München \\ Zentrum Mathematik \\ Garching bei München, Germany \\ karrasch@ma.tum.de
J. Triñanes*
Atlantic Oceanographic and Meteorological Laboratory National Oceanic \& Atmospheric
Administration
Miami, Florida, USA
joaquin.trinanes@noaa.gov

Started: September 23, 2021. This version: January 4, 2022.

\begin{abstract}
We identify effective carriers of Sargassum in the Caribbean Sea and describe a mechanism for coastal choking. Revealed from satellite altimetry, the carriers of Sargassum are mesoscale eddies (vortices of 50-km radius or larger) with coherent material (i.e., fluid) boundaries. These are observer-independent - unlike eddy boundaries identified with instantaneously closed streamlines of the altimetric sea-surface height field - and furthermore harbor finite-time attractors for networks of elastically connected finite-size buoyant or "inertial" particles dragged by ocean currents and winds,
\end{abstract}

*Also at Cooperative Institute for Marine \& Atmospheric Studies, University of Miami, Miami, Florida, USA and Departamento de Electrónica y Computación, Universidade de Santiago de Compostela, Santiago, Spain. 
a mathematical abstraction of Sargassum rafts. The mechanism of coastal inundation, identified using a minimal model of surface-intensified Caribbean Sea eddies, is thermal instability in the presence of bottom topography.

Keywords: Sargassum; Coherent Lagrangian vortex; Inertia; Thermal instability.

\section{Introduction}

Over this past decade, beaching events of pelagic Sargassum, a type of brown macroalgae that forms floating rafts at the ocean surface, have been reported nearly every spring and summer within the Caribbean Sea [Wang et al., 2019]. These rafts of algae serve as habitats for marine fauna [Bertola et al., 2020] and can be an important carbon sink with consequences for global climate regulation [Paraguay-Delgado et al., 2020]. At the same time, they can carry high levels of arsenic and heavy metals, producing major problems when decomposing on beaches such as negatively impacting seagrass communities, corals, and water quality with an increase in sea turtle and fish mortality, causing health problems in humans, diminishing tourism and, as a result, disrupting the local economy [Smetacek and Zingone, 2013; Resiere et al., 2018].

The negative consequences of Sargassum choking in the coasts of the Caribbean Sea requires improvement in forecasting of Sargassum beaching events to allow coastal zone managers and decision makers to timely prepare and respond adequately. This work contributes, in part, to fulfill this demand by unveiling especial ocean phenomena that have the ability to facilitate the transport of Sargassum that conduct to subsequent "waves" of coastal inundation. It also seeks to get a basic insight into the process leading to the latter.

The sequence of images in Fig. 1 corresponds to satellite-inferred Sargassum distribution on the surface of the ocean in the Caribbean Sea as obtained from the 7-day Floating Algae Density (FAD) [Trinãnes et al., 2021]. On any given day, the FAD represents the average percentage of Sargassum coverage within a unit area or an image pixel over the last seven days, ending on the given day. The FAD is computed from the Alternative Floating Algae Index (AFAI), which serves as a measure of the magnitude of MODIS (Moderate Resolution Imaging Spectroradiometer) red edge reflectance of floating vegetation [Wang and Hu, 2016]. Overlaid in yellow on each FAD field is a snapshot of the material (i.e., Lagrangian) boundary of an anticyclonic (i.e., clockwise rotating) mesoscale eddy, which we have named Kukulkan. Detected from satellite-altimetry measurements of sea-surface height (SSH) [Le Traon et al., 1998], the boundary of Kukulkan was found to experience literally no stretching from 15/May/2017 to 14/July/2017. However, despite the fact that the boundary of Kukulkan represents a barrier for fluid transport, on 26/Jun/2017 Sargassum is seen to spiral inward from the region surrounding Kukulkan to its interior, bypassing its boundary. While Kukulkan drifts westward, it carries Sargassum within. Eventually, as it encounters in its path shallower and shallower water, it destabilizes. This process is characterized by intense filamentation (reminiscent of a writhing sneak, as is Kukulkan - the Mesoamerican feathered serpent deity - commonly depicted). The filaments breaking away from Kukulkan, and the Sargassum carried within, reach the continental margins of Central America and eventually also the Yucatan Peninsula.

We dedicate the next section (2) to show that eddies with persistent coherent material boundaries such as Kukulkan - the carriers of Sargassum-commonly traverse the Caribbean Sea by building from incoherent fluid. This will be preceded by a review of the nonlinear dynamics technique [Haller and Beron-Vera, 2013, 2014] used to frame them. In Section 3 we articulate how "coherent Lagrangian eddies" have the capacity of capturing Sargassum rafts - the cargo - and of dragging them along. This will make use of recent results [Beron-Vera, 2021c] pertaining to the dynamics of finite-size or "inertial" particle motion on the air-sea interface under the combined action of ocean currents and winds. In Section 4 we discuss the mechanism that leads to Sargassum coastal inundation. This is based on the proposition of a minimal model for Caribbean Sea vortex dynamics, 


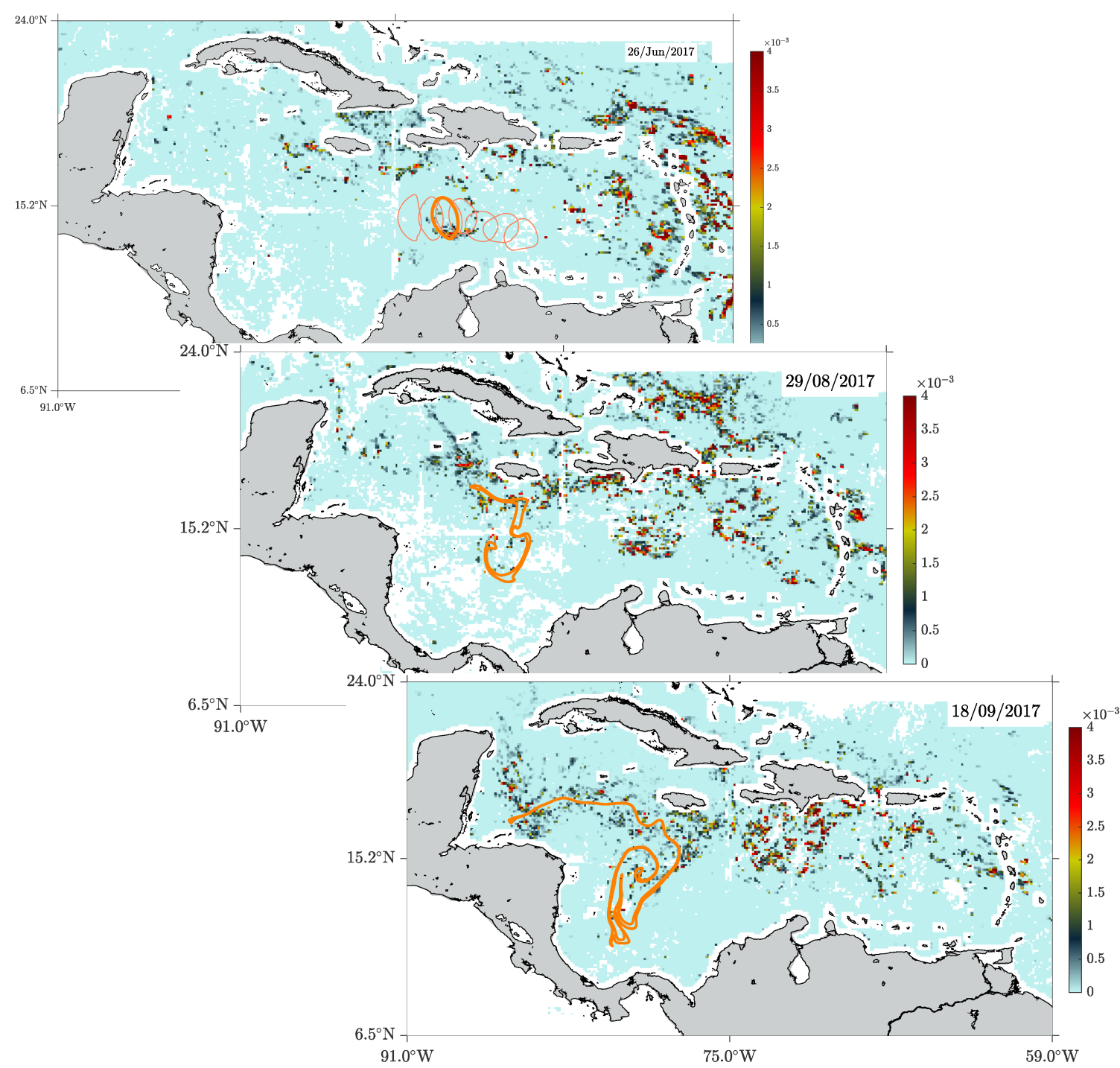

Figure 1: Sequence of images showing satellite-derived Sargassum distribution (percentage of coverage within a pixel) on the ocean surface in the Caribbean Sea. White represents absence of data. Overlaid on each image is the boundary of Kukulkan, an anticyclonic mesoscale eddy detected from satellite altimetry that was possible to be classified as coherent in a Lagrangian (i.e., fluid following) sense for two months. Forward and backward trailing advected images of the vortex boundary during that period, ranging from 15/May/2017 to 14/July/2017 (cf. Fig. 2), are depicted in thin in the top panel.

whose properties are discussed in the Appendix. The model builds on an old recipe, which used to be very common in ocean dynamics [Ripa, 1993] and is regaining momentum [Kurganov et al., 2020; Beron-Vera, 2021b,d,a; Holm et al., 2020], to include thermodynamics in the two-dimensional rotating shallow-water model. The paper is closed with a summary and some concluding remarks in Section 5. 


\section{The carriers}

Let $\mathbf{u}(\mathbf{x}, t)$ be a two-dimensional fluid velocity, with $\mathbf{x}$ denoting position in some domain of $\mathbb{R}^{2}$ and $t$ referring to time. Let $\varphi_{t_{0}}^{t}$ be the flow map associating fluid particle positions at times $t_{0}$ and $t$, which follows by integrating the motion equation, viz., $\dot{\mathbf{x}}=\mathbf{u}(\mathbf{x}, t)$.

The notion of a vortex with a material boundary resisting stretching under advection by the flow, e.g., inferred geostrophically from altimetry data, from time $t_{0}$ to time $t_{0}+T$ for some (finite) $T$ is expressed by the variational principle [Haller and Beron-Vera, 2013, 2014]

$$
\delta \oint \frac{\sqrt{\mathbf{r}^{\prime}(s) \cdot C_{t_{0}}^{t_{0}+T}(\mathbf{r}(s)) \mathbf{r}^{\prime}(s)}}{\sqrt{\mathbf{r}^{\prime}(s) \cdot \mathbf{r}^{\prime}(s)}} \mathrm{d} s=0 .
$$

Here, $\mathbf{r}(s)$ provides a parametrization for a material loop at time $t_{0}$ and $C_{t_{0}}^{t}:=\left(\mathrm{D} \varphi_{t_{0}}^{t}\right)^{\top} \mathrm{D} \varphi_{t_{0}}^{t}$, where $\mathrm{D}$ denotes derivative with respect to time- $t_{0}$ position, is the (symmetric, positive-definite) Cauchy-Green strain tensor field. The integrand in (1), which objectively (i.e., independent of the observer's viewpoint) measures relative stretching from $t_{0}$ to $t_{0}+T$, is symmetric under $s$-shifts and thus by Noether's theorem it must be equal to a positive constant, say $p$. In other words, solutions to (1) are characterized by uniformly $p$-stretching loops. The time- $t_{0}$ positions of such $p$-loops turn out to be limit cycles of one of the following two bidirectional vector or line fields:

$$
\mathbf{l}_{p}^{ \pm}(\mathbf{r}):=\sqrt{\frac{\lambda_{2}(\mathbf{r})-p^{2}}{\lambda_{2}(\mathbf{r})-\lambda_{1}(\mathbf{r})}} \mathbf{v}_{1}(\mathbf{r}) \pm \sqrt{\frac{p^{2}-\lambda_{1}(\mathbf{r})}{\lambda_{2}(\mathbf{r})-\lambda_{1}(\mathbf{r})}} \mathbf{v}_{2}(\mathbf{r})
$$

where $\lambda_{1}<p^{2}<\lambda_{2}$. Here, $\left\{\lambda_{i}\right\}$ and $\left\{\mathbf{v}_{i}\right\}$, satisfying $0<\lambda_{1} \leq \lambda_{2}, \mathbf{v}_{i} \cdot \mathbf{v}_{j}=\delta_{i j}, i, j=1,2$, are eigenvalues and (orientationless) normalized eigenvectors, respectively, of $C_{t_{0}}^{t_{0}+T}$. Limit cycles of (2) either grow or shrink under changes in $p$, forming smooth annular regions of nonintersecting loops. The outermost member of such a band of material loops is observed physically as the boundary of a coherent Lagrangian eddy, namely, a Lagrangian coherent structure [Haller, 2015] of elliptic type that generalizes the notion of KAM torus to the finite-time-aperiodic flow case [Haller and BeronVera, 2012]. The $p$-loops can also be interpreted as so-called null-geodesics of the (sign-indefinite) generalized Green-Lagrangian tensor field, $C_{t_{0}}^{t_{0}+T}-\lambda \mathrm{Id}$.

Remark 1 Two observations that follow from numerical experimentation [Andrade-Canto et al., 2020] are in order. First, the boundaries of coherent material vortices revealed from geodesic detection are not only resisting stretching, but also are nearly diffusion resisting [Haller et al., 2018]. Second, a stretching (or diffusion) withstanding Lagrangian eddy over $\left[t, t^{\prime}\right]$ typically includes, at $t$, a local maximum of the Lagrangian-averaged vorticity deviation (or LAVD), defined by [Haller et al., 2016$]$

$$
\operatorname{LAVD}_{t}^{t^{\prime}}(\mathbf{x}):=\int_{t}^{t^{\prime}}\left|\xi\left(\varphi_{t}^{\tau}(\mathbf{x}), \tau\right)-\bar{\xi}(\tau)\right| \mathrm{d} \tau
$$

where $\xi(\mathbf{x}, t)$ is the (vertical) vorticity of the fluid and $\bar{\xi}(t)$ is its average over the tracked fluid bulk.

In Fig. 2 we illustrate the full evolution of Kukulkan since its genesis by using the methodology devised by [Andrade-Canto et al., 2020]. This consists in repeatedly applying geodesic eddy detection on the (altimetric) flow domain of definition, exhaustively searching the two-parameter space $\left(t_{0}, T\right)$. More precisely, we roll the initial time instance $t_{0}$ over a time window covering the time interval of during which a vortex is expected to exist. For each $t_{0}$, we progress $T$ as long as the Lagrangian method successfully detects a coherent vortex. Thus, we obtain for each $t_{0}$ a life expectancy $T_{\max }\left(t_{0}\right)$, which is the maximum $T$ for which a Lagrangian simulation starting at $t_{0}$ successfully detected a coherent vortex. The expected results is a wedge-shaped $T_{\max }\left(t_{0}\right)$ distribution, indicating that all Lagrangian coherence assessments predict the breakdown consistently, independent of any 


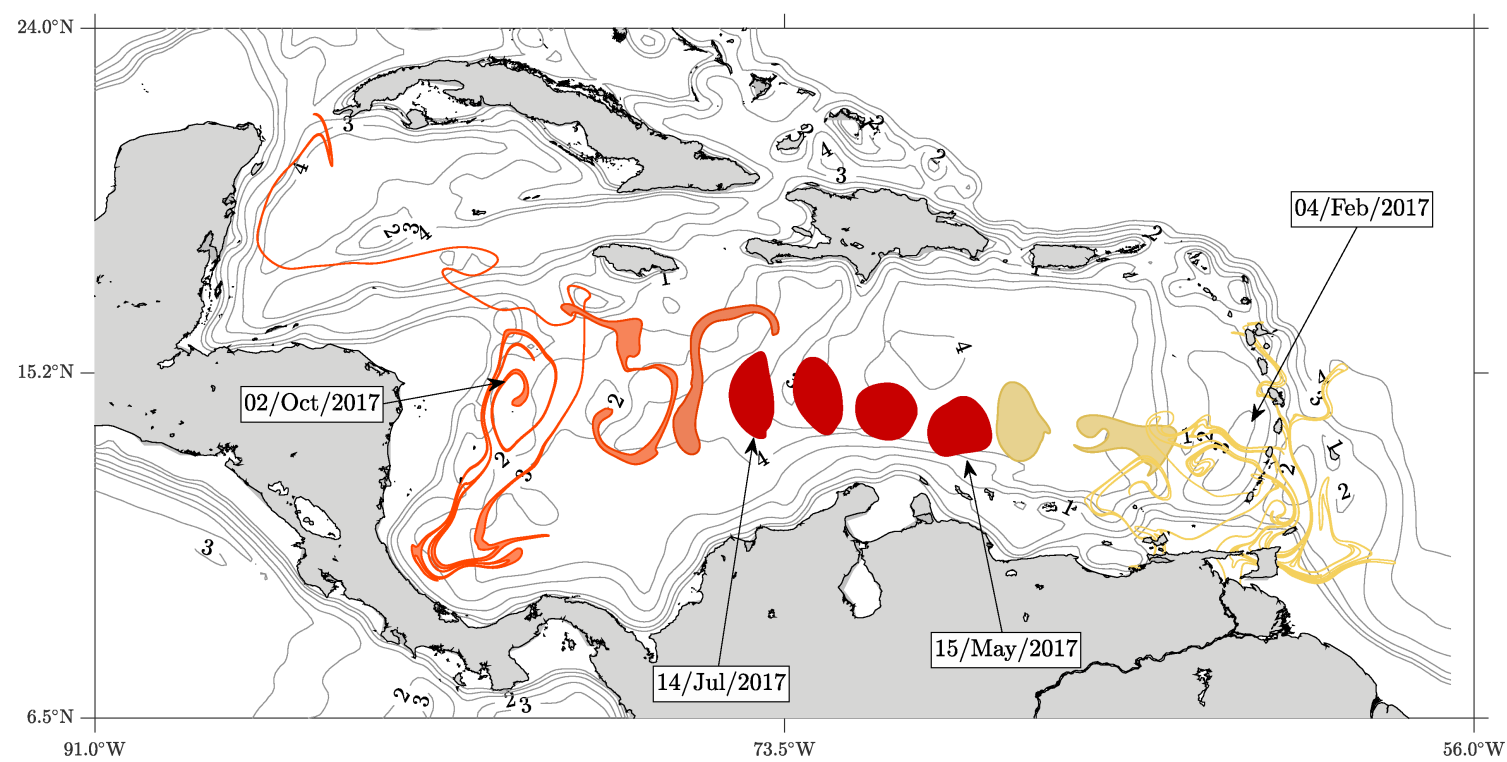

Figure 2: Genesis (yellow), evolution (red), and apocalypse (orange) of Kukulkan, an anticyclonic mesoscale vortex extracted from altimetry-derived surface geostrophic flow using geodesic detection. Selected isobaths (in $\mathrm{km}$ ) are shown in gray.

parameter presets. Robust assessments of the birth and decease dates of the vortex are obtained by combining the results from running the algorithm in forward- and backward-time directions. The detection scheme is numerically implemented in the Julia package CoherentStructures.jI by [Karrasch and Schilling, 2020] using the index theory for $p$-line fields (2) developed in [Karrasch et al., 2014]. As revealed in Fig. 2, Kukulkan builds material coherence out of fully incoherent fluid (water) that penetrates through the Lesser Antilles passages from the Atlantic Ocean. This happens on 15/May/2017.

Remark 2 While it is beyond the scope of this paper to explain the process that leads to the birth of Kukulkan and other eastern Caribbean Sea coherent Lagrangian eddies, this is certainly much more involved than the result of the squeezing of lens-like eddies (North Brazil Current rings) through gaps (Lesser Antilles passages) as envisioned by [Simmons and Nof, 2002]. Moreover, while eastern Caribbean Sea eddies can carry traces of Amazon and Orinoco Rivers water properties and sediments [van der Boog et al., 2019], recent claims [Huang et al., 2021] on the role of "SSH eddies" in connecting the tropical Atlantic Ocean and the Gulf of Mexico are unfounded. Identified as regions instantaneously encircled by SSH level curves, SSH eddies do not possess material boundaries. Thus, they cannot hold and carry within fluid long distances as coherent Lagrangian eddies to make connectivity assessments. The two main issues with this type of Eulerian eddy detection, which is the de-facto detection method in oceanography, are 1) its lack of objectivity, which leads to many false positives and also negatives [Beron-Vera et al., 2015], and, as discussed, 2) its lack of flow invariance.

Since its birth date, namely, the day in which it is first extracted from altimetry using geodesic detection, Kukulkan translates westward preserving its material coherence for nearly three months, in the rigorous sense that its boundary stretches by a factor $p=1.192$. (We use red color in Fig. 2 to depict the vortex while it is classified as Lagrangian coherent.) Eventually, starting on 14/July/2017, by the mechanism proposed below, Kukulkan loses its material coherence, spreading 


\begin{tabular}{lcccc}
\hline \hline Polarity & No. per year & $T[\mathrm{~d}]$ & $p$ & Mean radius $[\mathrm{km}]$ \\
Cyclonic & $2 \pm 2$ & $52 \pm 34$ & $1.23 \pm 0.33$ & $76 \pm 20$ \\
Anticyclonic & $4 \pm 3$ & $68 \pm 40$ & $1.23 \pm 0.34$ & $81 \pm 32$ \\
\hline
\end{tabular}

Table 1: Characteristic parameters of coherent Lagrangian eddies detected geodesically from altimetry over 2011-2019. Stated values are mean values, each one accompanied by a one-standard-deviation uncertainty.

its contents, mainly over the continental margins of Central America and the Yucatan Peninsula, along long filaments that break away from the vortex.

Since 2011, the year when the first major Sargassum event was recorded, there have been many other vortices traversing the eastern Caribbean Sea with a degree of material coherence similar to Kukulkan. We depict in Fig. 3 the trajectories of geodesically detected vortices from satellite altimetry over 2011-2019. Anticyclones (left panel) are as frequent as cyclones (right panel), which are smaller, have shorter lifetimes, and stretch more than anticyclones (Table 1).

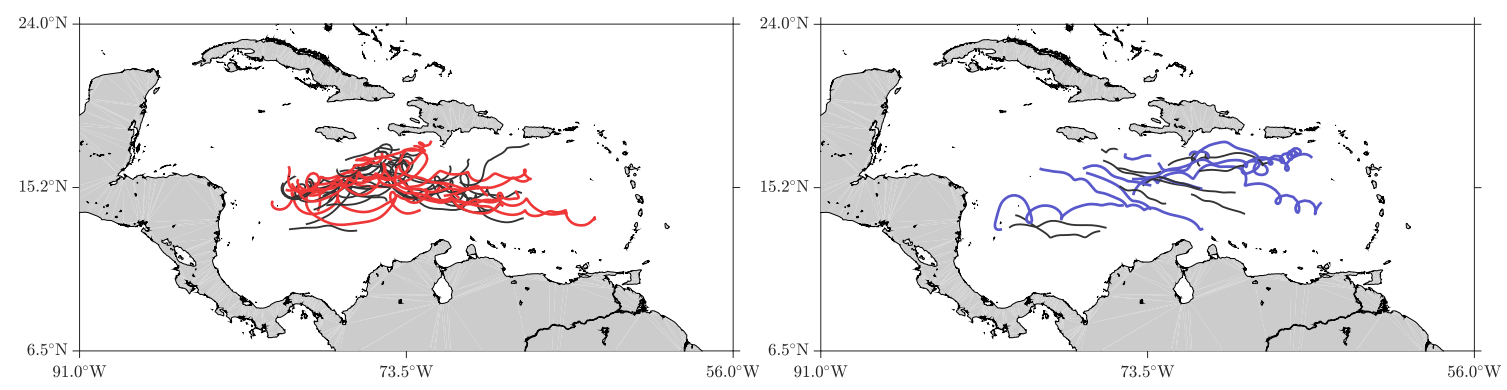

Figure 3: Trajectories of coherent Lagrangian eddies detected geodesically from altimetry over 2011-2019. Anticyclones (resp., cyclones) are depicted in the left (resp., right) panel. A gray trajectory indicates that the vortex was not seen to carry Sargassum or that the satellite imagery data were not sufficient to conclude it did.

\section{The cargo}

The notion that mesoscale vortices with material boundaries such as Kukulkan represent effective carriers of Sargassum, and, thus, through filamentation contribute to coastal inundation, is supported on the dynamics of elastically connected networks of finite-size buoyant or "inertial" particles [Beron-Vera and Miron, 2020], which are especial near so-called rotationally coherent vortices, i.e., with material boundaries given by convex level curves of the LAVD field (3) surrounding LAVD maxima [Haller et al., 2016]. Pelagic Sargassum rafts are composed of flexible stems kept afloat by gas-filled bladders. Elastic networks of inertial particles provide a minimal representation for them. Building on the Maxey-Riley equation for the dynamics of inertial particles floating at the air-sea surface of [Beron-Vera et al., 2019], referred to as the BOM equation, [Beron-Vera and Miron, 2020] show that the motion of an elastic network with $N$ inertial particles obeys

$$
\ddot{\mathbf{x}}_{i}+\left(\left.f\right|_{i}+\left.\frac{1}{3} \varrho \xi\right|_{i}\right) \dot{\mathbf{x}}_{i}^{\perp}+\frac{\dot{\mathbf{x}}_{i}}{\tau}=\left.\varrho \dot{\mathbf{u}}\right|_{i}+\left.\varrho\left(\left.f\right|_{i}+\left.\frac{1}{3} \xi\right|_{i}\right) \mathbf{u}\right|_{i} ^{\perp}+\frac{\left.(1-\alpha) \mathbf{u}\right|_{i}+\left.\alpha \mathbf{w}\right|_{i}}{\tau}+\mathbf{F}_{i}
$$


$i=1, \ldots, N$, where

$$
\mathbf{F}_{i}=-\sum_{j \in \operatorname{neighbor}(i)} k_{i j}\left(\left|\mathbf{x}_{i}-\mathbf{x}_{j}\right|-\ell_{i j}\right) \frac{\mathbf{x}_{i}-\mathbf{x}_{j}}{\left|\mathbf{x}_{i}-\mathbf{x}_{j}\right|}
$$

and $\left.\right|_{i}$ means pertaining to particle $i$. The system of coupled ordinary differential equations (4) represents a Newton law which includes, in addition to the forces included in the BOM equation (flow, added mass, lift, drag, and Coriolis), the elastic force (Hook's law) exerted on each particle by neighboring particles (4b), where $k_{i j}>0$ is the stiffness of the spring connecting particles $i$ and $j$ and $\ell_{i j}>0$ is the length of the latter at rest. The rest of the variables and parameters in (4a) is as follows: $\mathbf{x}_{i}(t)$ is the instantaneous position of particle $i ; \mathbf{u}(\mathbf{x}, t)$ is the near-surface ocean velocity, with $\dot{\mathbf{u}}(\mathbf{x}, t)$ denoting its total (material) derivative and $\xi(\mathbf{x}, t)$ its (vertical) vorticity; $\mathbf{w}(\mathbf{x}, t)$ is near-surface wind velocity; $f$ is the Coriolis parameter; and the parameters $\varrho \in[0,1)$, $\tau>0$ and $\alpha \in[0,1)$ depend on the water-to-particle density ratio $\delta \geq 1$ in specific forms as given in [Beron-Vera et al., 2019] [cf. also Olascoaga et al., 2020; Miron et al., 2020b,a]. In particular, $\tau$ measures the inertial response time of the medium to the particle, and is proportional to the square of the radius of the latter. In turn, $\alpha$ can be interpreted as a buoyancy-dependent leeway factor, terminology commonly used in the search-and-rescue-at-sea literature [Breivik et al., 2013]. An informal statement of Theorem 4.1 of [Beron-Vera and Miron, 2020] is as follows:

the trajectory of the center of a rotationally coherent vortex that spins anticyclonically (resp., cyclonically) is locally forward attracting over the lifetime of the vortex for all $k_{i j}$ (resp., if $\sum_{i=1}^{N} \sum_{j \in \text { neighbor }(i)} k_{i j}$ is larger than a quantity that decays with $N$ to a typically small value) provided that winds are sufficiently calm.

Moreover, numerical experimentation reveals that the above statement holds under fairly general wind conditions [Beron-Vera and Miron, 2020].

By view of the second observation in Remark 1, coherent Lagrangian vortices detected geodesically from altimetry-derived velocity should attract Sargassum rafts. This is consistent with Kukulkan collecting Sargassum, transporting it, and eventually, upon destabilization, spreading the Sargassum in the surrounding areas, which include the continental margins of the Yucatan Peninsula and Central America.

Figure 3 reveals that the behavior predicted by the theory of [Beron-Vera and Miron, 2020] applies to a large number of the coherent Lagrangian eddies travelling through the eastern Caribbean Sea. The trajectories of such vortices are indicated in color; the black lines correspond to trajectories of eddies that either did not capture Sargassum or the quality of the satellite imagery data was too poor (e.g., due to excessive cloud coverage) to determine that they did. More precisely, out of a total of 40 (resp., 21) geodesically detected anticyclones (resp., cyclones) over 2011-2019, 17 (resp., 10) were visually found to have Sargassum spiraling inward at some instant along their trajectories.

\section{The mechanism}

While in-situ sampling is not exhaustive, a recent hydrographic survey by [van der Boog et al., 2019] suggests that mesoscale anticyclones in the eastern Caribbean Sea are surface intensified. This is puzzling inasmuch as surface-intensified vortices should be able to relatively easily bypass topographic obstacles according to linear theory arguments and fully nonlinear numerical simulations by [Adams and Flierl, 2010]. This assessment, however, was based on the consideration of a model with two homogeneous density layers. We explore here how this scenario changes by making such a model more realistic through the incorporation of density inhomogeneity effects. This is done in the simplest manner possible, by allowing the density (temperature) in the upper layer to vary laterally and with time, while keeping it as well as the (horizontal) velocity as depth independent [e.g., Ripa, 1993]. 
Our minimal two-layer model is formulated as follows. The upper layer has, as anticipated, density varying in lateral position, $\mathbf{x}=(x, y)$, and time, $t$, and is limited from above by a horizontal rigid lid. Position $\mathbf{x}$ is assumed to range on a domain $D$ of the $\beta$ plane, so $x$ (resp., $y$ ) points eastward (resp., northward). Specifically, $D=[0,30 R] \times[0,10 R]$, centered in the eastern Caribbean Sea, where

$$
R^{2}:=\frac{g^{\prime} H / f_{0}^{2}}{1+r^{-1}}
$$

is the square of the Rossby radius of deformation. Here, $f_{0}>0$ is the mean Coriolis parameter in $D ; g^{\prime}>0$ is the buoyancy ${ }^{1}$ jump across the interface between the upper and lower layers in the reference state, i.e., with constant density in the upper layer and no currents in either layer; $H>0$ is the mean thickness of the lower layer; and $r H, r>0$, is the mean thickness of the upper layer. From the global climatological data [Chelton et al., 1998] it follows that $R=60 \mathrm{~km}$ is representative of eastern Caribbean Sea conditions. This justifies our domain size choice. From [van der Boog et al., 2019] in-situ hydrographic observations, $r=0.2$ seems fair (the depth in the eastern Caribbean Sea can reach $5 \mathrm{~km}$ or more; cf. Fig. 2). The lower layer has homogeneous density, is (much) heavier than the upper layer, and rests on a rigid bottom with at most a constant zonal slope $-H \beta_{\mathrm{T}} / f_{0}$, where $\beta_{\mathrm{T}}>0$ is a constant. Assuming quasigeostrophic (QG) dynamics, the model equations take the form

$$
\left.\begin{array}{rl}
\partial_{t} q_{i}+\left[\psi_{i}, q_{i}\right]-\delta_{1 i} R^{-2}\left[\psi_{1}, \psi\right] & =0, \\
\partial_{t} \psi+\left[\psi_{1}, \psi\right] & =0,
\end{array}\right\}
$$

$i=1,2$. Here, $[A, B]:=\nabla^{\perp} A \cdot \nabla B$ is the Jacobian for (time-dependent) functions $A$ and $B$ on $\mathbb{R}^{2}$, and the upper $(i=1)$ and lower $(i=2)$ layer streamfunctions $\left(\psi_{1}(\mathbf{x}, t), \psi_{2}(\mathbf{x}, t)\right)$ are related to the fields $\left(q_{1}(\mathbf{x}, t), \psi(\mathbf{x}, t), q_{2}(\mathbf{x}, t)\right)$ through the invertibility principle

$$
\nabla^{2} \psi_{i}+\frac{(-1)^{i} R^{-2}}{1+r^{(-1)^{i+1}}}\left(\psi_{1}-\psi_{2}\right)=q_{i}-\delta_{1 i} R^{-2} \psi-\beta y-\delta_{2 i}(1-\theta(x-15 R)) \beta_{\mathrm{T}} x
$$

where $\theta$ is the Heaviside step function. Note that the topographic slope is nonzero eastward of the mid zonal point of the domain, minimally representing the actual topography in the eastern Caribbean Sea. This is roughly achieved by setting $\beta_{\mathrm{T}}=0.005 f_{0} / H$. The field $q_{i}$ represents the $i$ th-layer QG potential vorticity and $2 g^{\prime} \psi / f_{0} R^{2}$ is the QG buoyancy deviation from the reference buoyancy $\left(g^{\prime}\right)$ in the upper layer. The subspace $\{\psi=$ const $\}$ is invariant. The dynamics on it are governed by the QG model with two homogeneous layers [e.g., Ripa, 1991]. Setting $\psi_{2}=0$ and making $H \rightarrow \infty$ while keeping $r H$ finite, leads to the inhomogeneous-layer reduced-gravity QG model originally developed in [Ripa, 1996]. See [Beron-Vera, 2021d,a; Holm et al., 2020] for recent discussions on geometric aspects of this model as well as on sustained "thermal" instabilities [Gouzien et al., 2017], and [Crisan et al., 2021] for the construction of unique solutions. Well-posedness of (6) is here supported on its uniqueness of solutions and manifest generalized (noncanonical) Hamiltonian structure (cf. Appendix).

The simulations are initialized by specifying $\psi_{i}$ and $\psi$. Specifically, we set

$$
\psi_{i}(\mathbf{x}, 0)=\sqrt{\mathrm{e}} R V_{i} \mathrm{e}^{\frac{-(x-27 R)^{2}-(y-7 R)^{2}}{2 R^{2}}},
$$

with $V_{1}=0.75 \mathrm{~m} \mathrm{~s}^{-1}$, supported on [van der Boog et al., 2019] in-situ hydrographic measurements. This represents an initially localized vortex-like structure with anticyclonic polarity. Without observational support we choose $V_{2}=V_{1} / 4$, yet consistent with earlier characterizations of surfaceintensified vortices [Adams and Flierl, 2010], and $\psi(\mathbf{x}, 0)=\psi_{2}(\mathbf{x}, 0)$. We also impose a background westward flow in upper layer of $0.1 \mathrm{~m} \mathrm{~s}^{-1}$, estimated from the time coherent Lagrangian eddies

${ }^{1}$ The buoyancy is gravity times the ratio of the difference between the lower and upper layer densities to the (constant) Boussinesq approximation's density. 
detected from altimetry typically take to traverse the eastern Caribbean Sea. This is on the order of the mean zonal velocity of the Caribbean Current based on satellite-tracked surface drifter trajectories [Richardson, 2005]. We use the same background westward velocity in the lower layer to minimize the development of baroclinic instability. This choice is not an artifact, but is actually supported by the observation, presented in the preceding section, that eastern Caribbean Sea eddies can remain materially coherent for months. The results are not sensitive to the other parameter choices.
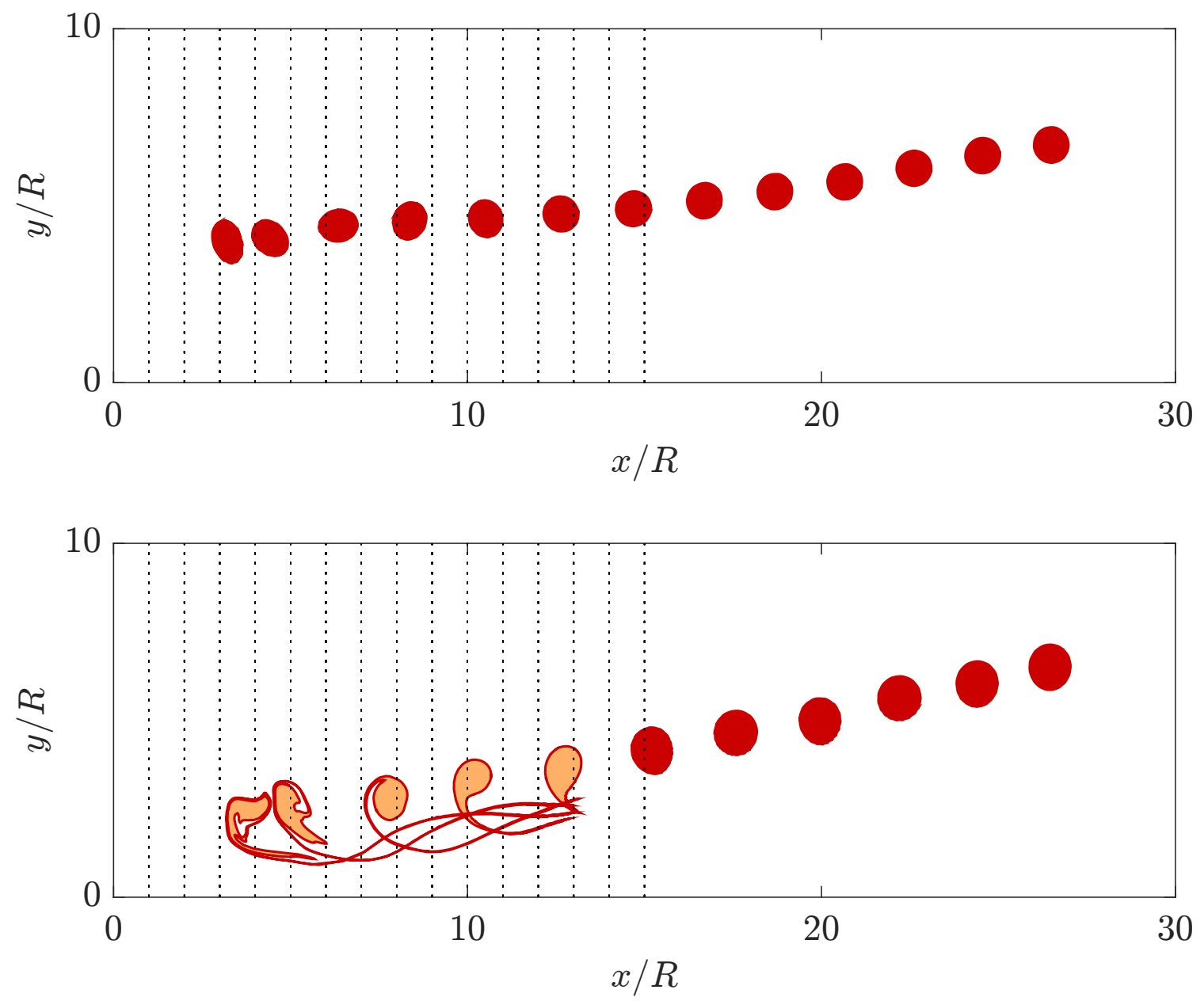

Figure 4: Snapshots reflecting the evolution every $15 \mathrm{~d}$ of a surface-intensified Lagrangian vortex classified as coherent by geodesic detection over the period that the structure is depicted in red, according to the quasigeostrophic two-layer model (6) with homogeneous (top) and inhomogeneous (bottom) density in the upper layer. Broken lines indicate isobaths, with depth decreasing linearly to the west (left). Length is scaled by the (internal) Rossby deformation radius.

The results strongly depend, however, on whether density inhomogeneity (in the upper layer) is allowed or not. This is illustrated in Fig. 4, with each panel showing snapshots, every $15 \mathrm{~d}$, of the westward translation of a structure revealed as a coherent Lagrangian vortex using geodesic detection. The upper (resp., lower) panel corresponds to a simulation with homogeneous (resp., 
inhomogeneous) density. The simulations were performed on $D=\mathbb{R} / 30 R \mathbb{Z} \times \mathbb{R} / 10 R \mathbb{Z}$, for simplicity as commonly done [Adams and Flierl, 2010], using a fully-dealiased pseudospectral code on a $512 \times 256$ grid with a fourth-order Runge-Kutta time stepper for $\left(q_{1}-\beta y, \psi, q_{2}-\beta y-\beta_{\mathrm{T}} x\right)$ and bi-Laplacian hyperviscosity [cf. Beron-Vera et al., 2008, for details]. In each panel the first red blob to the right (east) corresponds to the vortex at the instance it first acquires Lagrangian coherence. This happens approximately $5 \mathrm{~d}$ after initialization at $\mathbf{x}=(27 R, 7 R)$ for the simulation without inhomogeneous density and with it. Both the homogeneous and inhomogeneous vortex take a southwestward trajectory, consistent with numerical simulations and rotating-tank experiments [Carnevale et al., 1991] and altimetry observations [Morrow et al., 2004]. We depict in red the advected images of each vortex while classified as Lagrangian coherent, and in orange the advected images past the Lagrangian coherence horizon. The homogeneous vortex remains coherent, in the most strict geodesic sense with stretching factor $p \approx 1$, for the extent of the simulation, $145 \mathrm{~d}$, while the inhomogeneous vortex for only $90 \mathrm{~d}$. The condition $p \approx 1$ and area preservation (which follows from $\nabla \cdot \nabla^{\perp} \psi_{1}=0$ ) impose a strong restriction on the deformation of these vortices respectively over $t \in[5,150] \mathrm{d}$ and $t \in[5,95] \mathrm{d}$, as is evident from Fig. 4. The homogeneous vortex is much more longevous than the inhomogeneous one. It bypasses the topographic slope, consistent with earlier predictions [Adams and Flierl, 2010]. The only evident effect on the vortex is a slight change in its trajectory. In stark contrast, the inhomogeneous vortex experiences vigorous filamentation as soon as it starts to travel over shallower fluid (the vertical broken isolines indicate isobaths).

Our minimal model of surface-intensified Caribbean Sea vortex dynamics thus identifies thermal instability in the presence of bottom topography as a mechanism for vortex filamentation. This is consequential for Sargassum coastal inundation as articulated in the previous sections.

Remark 3 Self-induced westward propagation [Nof, 1981] is hard to realize in the simulations. On the other hand, if these are initialized with a cyclonic vortex structure, the trajectories result northwestward, consistent with the numerical, experimental, and observational evidence given in [Carnevale et al., 1991; Morrow et al., 2004]. The eastern Caribbean Sea, by contrast with our minimal model simulations, seems too constrained in longitude for coherent Lagrangian vortices detected from altimetry to clearly reveal a preferred meridional direction in their westward drift. Only cyclones reveal a predominantly northwestward translation pattern (cf. Fig. 3). The inhomogeneous vortex takes $125 d$ to cover the same longitudinal distance that the homogeneous vortex covers in $145 \mathrm{~d}$. Inhomogeneity thus contributes to speeding up the vortex translation. Finally, that material coherence can be realized for the noted extended periods of time, particularly in the homogeneous case, is surprising inasmuch as (7) does not represent a solution of (6) and Rossby-wave radiation invariably happens immediately after initialization [e.g. Adams and Flierl, 2010].

\section{$5 \quad$ Summary and concluding remarks}

We have shown that Sargassum transport in the eastern Caribbean Sea can be effectively accomplished by coherent Lagrangian mesoscale eddies. Geodesically detected from satellite altimetry, these vortices, despite having material boundaries that resist stretching and are impassable by fluid particles, contain finite-time attractors for Sargassum. This was confirmed by satellite observations in agreement with the prediction by the Maxey-Riley theory for the motion of elastic networks of finite-size particles floating at the ocean surface. The coastal inundation of Sargassum was found to be associated with the filamentation that the vortices experience as they encounter shallower water in their westward drift. Thermal instability of surface-intensified vortices interacting with topography was identified as a mechanism leading to the filamentation. This was done by proposing a minimal model for (eastern) Caribbean Sea vortex dynamics with solid geometric properties that revisits a simple recipe, once very popular, then abandoned, and now experiencing a strong comeback, to incorporate thermodynamics in a shallow-water model. Our results are consequential 
for the prediction of Sargassum inundation events, and thus for planning an adequate response to them. The basic take-home message is:

to aid in the forecasting of Sargassum coastal arrivals, monitor the progression of mesoscale eddies geodesically detected from altimetry.

This might seem an impossible task as it appears to require unavailable future altimetry-derived flow information. However, geodesic eddy detection can be applied in backward time, as shown in [Andrade-Canto et al., 2020]. In other words, material coherence assessments can be made with observed velocity up to the assessment instant. Finally, from a theoretical view point, substantial work, beyond the scope of the analysis tools used here, needs to be done to understand the process that leads to the formation of coherent material vortices, particularly eastern Caribbean Sea vortices, out of incoherent fluid. Thus far this remains a mystery.

\section{Acknowledgements}

This work was supported by the University of Miami's Cooperative Institute for Marine \& Atmospheric Studies.

\section{Author declarations}

\section{Conflict of interest}

The authors have no conflicts to disclose.

\section{Data availability}

This paper does not involve the use of data. The floating algae density fields are produced by USF and distributed by SaWS (https://optics.marine.usf.edu/projects/saws.html). The altimeter products are produced by SSALTO/DUCAS and distributed by AVISO with support from CNES (http://www.aviso.oceanobs).

\section{A Uniqueness of solutions and Hamiltonian structure of (6)}

Let $\varphi_{i}:=q_{i}-\delta_{1 i} R^{-2} \psi-\beta y-\delta_{2 i}(1-\theta(x-10 R)) \beta_{\mathrm{T}} x$. Writing $\psi_{i}=(-1)^{i} r^{i-2} \hat{\psi}_{1}+\hat{\psi}_{2}$ and similarly for $\varphi_{i}$, from $(6 \mathrm{~b})$ one finds

$$
\nabla^{2} \hat{\psi}_{i}-\delta_{1 i} R^{-2} \hat{\psi}_{i}=\hat{\varphi}_{i} .
$$

Assume that $\hat{\psi}_{i}^{(1)}$ and $\hat{\psi}_{i}^{(2)}$ satisfy (8) for $i=1,2$ on $D \subseteq \mathbb{R}^{2}$ under appropriate boundary conditions. These are: 1) $\nabla \hat{\psi}_{i}^{(j)} \times\left.\hat{\mathbf{n}}\right|_{\partial D}=0$ where $\hat{\mathbf{n}}$ is the unit normal to the solid boundary of $\left.D ; 2\right) \hat{\psi}_{i}^{(j)} \rightarrow 0$ as $\mathbf{x} \rightarrow \infty$ if $D$ spans $\mathbb{R}^{2}$, or 3$) \hat{\psi}_{i}^{(j)}\left(x+L_{x}\right)=\hat{\psi}_{i}^{(j)}$ and $\hat{\psi}_{i}^{(j)}\left(y+L_{y}\right)=\hat{\psi}_{i}^{(j)}$ when $D=\mathbb{R} / L_{x} \mathbb{Z} \times$ $\mathbb{R} / L_{y} \mathbb{Z}$. Eliminating $\hat{\varphi}_{j}$ from

$$
\nabla^{2} \hat{\psi}_{i}^{(j)}-\delta_{1 i} R^{-2} \hat{\psi}_{i}^{(j)}=\hat{\varphi}_{i}
$$

multiplying the result by $\psi_{i}^{(1)}-\psi_{i}^{(2)}$, and integrating by parts,

$$
\int_{D}\left|\nabla\left(\psi_{i}^{(1)}-\psi_{i}^{(2)}\right)\right|^{2}+\delta_{1 i} R^{-2}\left(\psi_{i}^{(1)}-\psi_{i}^{(2)}\right)^{2} \mathrm{~d} x \mathrm{~d} y=0,
$$

from which it follows that $\hat{\psi}_{i}^{(1)}=\hat{\psi}_{i}^{(2)}$. Thus if a solution to (6) exists, then it is unique.

Now let $\mathscr{U}[\mu]=\int_{D} U\left(\mathbf{x}, \mu, \partial_{x} \mu, \partial_{y} \mu, \partial_{x y} \mu, \ldots\right) \mathrm{d} x \mathrm{~d} y$ be a functional of sufficiently smooth fields $\mu(\mathbf{x})=\left(\mu^{1}(\mathbf{x}), \mu^{2}(\mathbf{x}), \ldots\right)$ on $D$, which will be assumed to either be bounded by a solid boundary 
or span $\mathbb{R}^{2}$. The functional derivative of $\mathscr{U}$ with respect to $\mu^{a}$, denoted $\frac{\delta \mathscr{U}}{\delta \mu^{a}}$, is the unique element satisfying $\mathscr{U}\left[\mu^{a}+\varepsilon \delta \mu^{a}\right]-\mathscr{U}\left[\mu^{a}\right]=\varepsilon \int_{D} \frac{\delta \mathscr{U}}{\delta \mu^{a}} \delta \mu^{a} \mathrm{~d} x \mathrm{~d} y+O\left(\varepsilon^{2}\right)$ as $\varepsilon \rightarrow 0$. We will say that $\mathscr{U}[\mu]$ is admissible if $\left.\nabla \frac{\delta \mathscr{U}}{\delta \mu^{a}} \cdot \hat{\mathbf{n}}\right|_{\partial D}=0$ or $\frac{\delta \mathscr{U}}{\delta \mu^{a}} \rightarrow 0$ as $\mathbf{x} \rightarrow \infty$. The set of admissible functionals, denoted $\mathcal{A}$, cannot be extended to functionals of functions on $D=\mathbb{R} / L_{x} \mathbb{Z} \times \mathbb{R} / L_{y} \mathbb{Z}$. With this in mind, (6) can be cast as a generalized (noncanonical) Hamiltonian system [Morrison, 1998]

$$
\partial_{t} \mu=\{\mu, \mathscr{H}\}
$$

for $\mu:=\left(q_{1}, \psi, q_{2}\right)$, with Hamiltonian given by

$$
\mathscr{H}[\mu]:=\frac{1}{2} \int_{D} H_{1}\left|\nabla \psi_{1}\right|^{2}+H_{2}\left|\nabla \psi_{2}\right|^{2}+\frac{f_{0}^{2}}{g^{\prime}}\left(\psi_{1}-\psi_{2}\right)^{2} \mathrm{~d} x \mathrm{~d} y
$$

and Lie-Poisson bracket [Thiffeault and Morrison, 2000]

$$
\{\mathscr{U}, \mathscr{V}\}[\mu]:=W_{c}^{a b} \int_{D} \mu^{c}\left[\frac{\delta \mathscr{U}}{\delta \mu^{a}} \frac{\delta \mathscr{V}}{\delta \mu^{b}}\right] \mathrm{d} x \mathrm{~d} y
$$

for all $\mathscr{U}, \mathscr{V}[\mu] \in \mathcal{A},{ }^{2}$ where the $(2,1)$-tensor $W$ has coefficients $W_{1}^{11}=W_{2}^{12}=W_{2}^{21}=(r H)^{-1}$, $W_{3}^{33}=H^{-1}$, and zero otherwise. That (13) represents a genuine bracket follows from $W_{c}^{a b}=W_{c}^{b a}$ and $W_{c}^{a b} W_{a}^{a^{\prime} b^{\prime}}=W_{c}^{a b^{\prime}} W_{a}^{b^{\prime} a^{\prime}}$, which respectively imply antisymmetry for the bracket $(\{\mathscr{U}, \mathscr{V}\}=$ $-\{\mathscr{V}, \mathscr{U}\}$ for $\mathscr{U}, \mathscr{V}[\mu] \in \mathcal{A})$ and guarantee that it satisfies the Jacobi identity $(\{\{\mathscr{U}, \mathscr{V}\}, \mathscr{W}\}+$ $\{\{\mathscr{W}, \mathscr{U}\}, \mathscr{V}\}+\{\{\mathscr{V}, \mathscr{W}\}, \mathscr{U}\}=0$ for $\mathscr{U}, \mathscr{V}, \mathscr{W}[\mu] \in \mathcal{A})$. The associated infinite-family of invariant Casimirs is given by $\mathscr{C}[\mu]=\int q_{1} C_{1}(\psi)+C_{2}(\psi)+C_{3}\left(q_{2}\right)$ for any $C_{i}$, which commutes in the bracket with any $\mathscr{U}[\mu] \in \mathcal{A}$.

Remark 4 The geometry of (13) is most easily grasped by writing it explicitly as

$$
\{\mathscr{U}, \mathscr{V}\}=\int_{D} \frac{q_{1}}{r H}\left[\frac{\delta \mathscr{U}}{\delta q_{1}}, \frac{\delta \mathscr{V}}{\delta q_{1}}\right]+\frac{\psi}{r H}\left(\left[\frac{\delta \mathscr{U}}{\delta q_{1}}, \frac{\delta \mathscr{V}}{\delta \psi}\right]-\left[\frac{\delta \mathscr{V}}{\delta q_{1}}, \frac{\delta \mathscr{U}}{\delta \psi}\right]\right)+\frac{q_{2}}{H}\left[\frac{\delta \mathscr{U}}{\delta q_{2}}, \frac{\delta \mathscr{V}}{\delta q_{2}}\right] \mathrm{d} x \mathrm{~d} y
$$

Let $\mathfrak{a}$ be the Lie enveloping algebra of $\operatorname{SDiff}(D)$, the group of area preserving diffeomorphisms in $D$. The corresponding vector space is that of smooth time-dependent functions in $D$, denoted $\mathcal{F}(D)$, and the Lie bracket is given by the canonical Poisson bracket, [, ]. The Lie-Poisson bracket (13) represents a product for a realization of a Lie enveloping algebra on functionals in the dual (with respect to the $\mathrm{L}_{2}$ inner product) of $\mathfrak{a} \times \mathfrak{a}_{\mathrm{s}}$, where $\mathfrak{a}_{\mathrm{s}}$ is the extension of $\mathfrak{a}$ by semidirect sum to the vector space $\mathfrak{a} \times \mathcal{F}(D)$, with the representation of $\mathfrak{a}$ on $\mathcal{F}(D)$ given by [,] [cf. Thiffeault and Morrison, 2000, for details].

\section{References}

Adams, D. K. and Flierl, G. R. [2010]. Modeled interactions of mesoscale eddies with the East Pacific Rise: Implications for larval dispersal. Deep Sea Research Part I: Oceanographic Research Papers 57, 1163-1176.

Andrade-Canto, F., Karrasch, D. and Beron-Vera, F. J. [2020]. Genesis, evolution, and apocalyse of Loop Current rings. Phys. Fluids 32, 116603.

Beron-Vera, F. J. [2021a]. Extended shallow-water theories with thermodynamics and geometry. Phys. Fluids 33, 106605.

Beron-Vera, F. J. [2021b]. Multilayer shallow-water model with stratification and shear. Rev. Mex. Fis. 67, 351-364.

\footnotetext{
${ }^{2}$ We are implicitly assuming that $\mathcal{A}$ is closed, namely, if $\mathscr{U}, \mathscr{V}[\mu] \in \mathcal{A}$, then $\{\mathscr{U}, \mathscr{V}\}[\mu] \in \mathcal{A}$.
} 
Beron-Vera, F. J. [2021c]. Nonlinear dynamics of inertial particles in the ocean: From drifters and floats to marine debris and Sargassum. Nonlinear Dyn. 103, 1-26.

Beron-Vera, F. J. [2021d]. Nonlinear saturation of thermal instabilities. Phys. Fluid 33, 036608.

Beron-Vera, F. J., Brown, M. G., Olascoaga, M. J., Rypina, I. I., Kocak, H. and Udovydchenkov, I. A. [2008]. Zonal jets as transport barriers in planetary atmospheres. J. Atmos. Sci. 65, 33163326 .

Beron-Vera, F. J. and Miron, P. [2020]. A minimal Maxey-Riley model for the drift of Sargassum rafts. J. Fluid Mech. 904, A8.

Beron-Vera, F. J., Olascoaga, M. J., Haller, G., Farazmand, M., Triñanes, J. and Wang, Y. [2015]. Dissipative inertial transport patterns near coherent Lagrangian eddies in the ocean. Chaos 25, 087412.

Beron-Vera, F. J., Olascoaga, M. J. and Miron, P. [2019]. Building a Maxey-Riley framework for surface ocean inertial particle dynamics. Phys. Fluids 31, 096602.

Bertola, L. D., Boehm, J. T., Putman, N. F., Xue, A. T., Robinson, J. D., Harris, S., Baldwin, C. C., Overcast, I. and Hickerson, M. J. [2020]. Asymmetrical gene flow in five co-distributed syngnathids explained by ocean currents and rafting propensity. Proceedings of the Royal Society B 287, 20200657.

van der Boog, C. G., de Jong, M. F., Scheidat, M., Leopold, M. F., Geelhoed, S. C. V., Schulz, K., Dijkstra, H. A., Pietrzak, J. D. and Katsman, C. A. [2019]. Hydrographic and biological survey of a surface-intensified anticyclonic eddy in the Caribbean Sea. Journal of Geophysical Research 124 (8), 6235-6251.

Breivik, O., Allen, A. A., Maisondieu, C. and Olagnon, M. [2013]. Advances in search and rescue at sea. Ocean Dynamics 63, 83-88.

Carnevale, G. F., Kloosterziel, R. C. and van Heist, G. J. F. [1991]. Propagation of barotropic vortices over topography in a rotating tank. J. Fluid Mech. 255, 119-139.

Chelton, D. B., deSzoeke, R. A., Schlax, M. G., El Naggar, K. and Siwertz, N. [1998]. Geographical variability of the first baroclinic rossby radius of deformation. J. Phys. Oceanogr. 28, 433-460.

Crisan, D., Holm, D. D., Luesink, E., Mensah, P. R. and Pan, W. [2021]. Theoretical and computational analysis of the thermal quasi-geostrophic model. arXiv:2106.14850.

Gouzien, E., Lahaye, N., Zeitlin, V. and Dubos, T. [2017]. Thermal instability in rotating shallow water with horizontal temperature/density gradients. Physics of Fluids 29, 101702.

Haller, G. [2015]. Lagrangian coherent structures. Ann. Rev. Fluid Mech. 47, 137-162.

Haller, G. and Beron-Vera, F. J. [2012]. Geodesic theory of transport barriers in two-dimensional flows. Physica D 241, 1680-1702.

Haller, G. and Beron-Vera, F. J. [2013]. Coherent Lagrangian vortices: The black holes of turbulence. J. Fluid Mech. 731, R4.

Haller, G. and Beron-Vera, F. J. [2014]. Addendum to 'Coherent Lagrangian vortices: The black holes of turbulence'. J. Fluid Mech. 755, R3.

Haller, G., Hadjighasem, A., Farazmand, M. and Huhn, F. [2016]. Defining coherent vortices objectively from the vorticity. J. Fluid Mech. 795, 136-173.

Haller, G., Karrasch, D. and Kogelbauer, F. [2018]. Material barriers to diffusive and stochastic transport. Proceedings of the National Academy of Sciences 115, 9074-9079.

Holm, D. D., Luesink, E. and Pan, W. [2020]. Stochastic mesoscale circulation dynamics in the thermal ocean. Phys. Fluids 33, 046603.

Huang, M., Liang, X., Zhu, Y., Liu, Y. and Weisberg, R. H. [2021]. Eddies connect the tropical Atlantic Ocean and the Gulf of Mexico. Geophysical Research Letters 48, e2020GL091277.

Karrasch, D., Huhn, F. and Haller, G. [2014]. Automated detection of coherent Lagrangian vortices in two-dimensional unsteady flows. Proc. Royal Soc. A 471, 20140639.

Karrasch, D. and Schilling, N. [2020]. Fast and robust computation of coherent lagrangian vortices on very large two-dimensional domains. The SMAI journal of computational mathematics 6 , $101-124$. 
Kurganov, A., Liu, Y. and Zeitlin, V. [2020]. Moist-convective thermal rotating shallow water model. Physics of Fluids 32 (6), 066601.

Le Traon, P. Y., Nadal, F. and Ducet, N. [1998]. An improved mapping method of multisatellite altimeter data. J. Atmos. Oceanic Technol. 15, 522-534.

Miron, P., Medina, S., Olascaoaga, M. J. and Beron-Vera, F. J. [2020a]. Laboratory verification of a Maxey-Riley theory for inertial ocean dynamics. Phys. Fluids 32, 071703.

Miron, P., Olascoaga, M. J., Beron-Vera, F. J., Triñanes, J., Putman, N. F., Lumpkin, R. and Goni, G. J. [2020b]. Clustering of marine-debris-and Sargassum-like drifters explained by inertial particle dynamics. Geophys. Res. Lett. 47, e2020GL089874.

Morrison, P. J. [1998]. Hamiltonian description of the ideal fluid. Rev. Mod. Phys. 70, 467-521.

Morrow, R., Birol, F. and Griffin, D. [2004]. Divergent pathways of cyclonic and anti-cyclonic ocean eddies. Geophys. Res. Lett. 31, L24311.

Nof, D. [1981]. On the $\beta$-induced movement of isolated baroclinic eddies. J. Phys. Oceanogr. 11, $1662-1672$.

Olascoaga, M. J., Beron-Vera, F. J., Miron, P., Triñanes, J., Putman, N. F., Lumpkin, R. and Goni, G. J. [2020]. Observation and quantification of inertial effects on the drift of floating objects at the ocean surface. Phys. Fluids 32, 026601.

Paraguay-Delgado, F., Carreno-Gallardo, C., Estrada-Guel, I., Zabala-Arceo, A., MartinezRodriguez, H. A. and Lardizabal-Gutierre, D. [2020]. Pelagic Sargassum spp. capture $\mathrm{CO}_{2}$ and produce calcite. Environ Sci. Pollut. Res. 42, https://doi.org/10.1007/s11356-020-08969-w.

Resiere, D., Valentino, R., Neviere, R., Banydeen, R., Gueye, P., Florentin, J., Cabie, A., Lebrun, T., Megarbane, B., Guerrier, G. and Mehdaoui, H. [2018]. Sargassum seaweed on Caribbean islands: an international public health concern. The Lancet 392, 2691.

Richardson, P. [2005]. Caribbean Current and eddies as observed by surface drifters. Deep Sea Research Part II: Topical Studies in Oceanography 52, 429-463.

Ripa, P. [1991]. General stability conditions for a multi-layer model. J. Fluid Mech. 222, 119-137.

Ripa, P. [1993]. Conservation laws for primitive equations models with inhomogeneous layers. Geophys. Astrophys. Fluid Dyn. 70, 85-111.

Ripa, P. [1996]. Low frequency approximation of a vertically integrated ocean model with thermodynamics. Rev. Mex. Fis. 42, 117-135.

Simmons, H. and Nof, D. [2002]. The squeezing of eddies through gaps. J. Phys. Oceanogr. 32, $314-335$.

Smetacek, V. and Zingone, A. [2013]. Green and golden seaweed tides on the rise. Nature 504, $84-88$.

Thiffeault, J.-L. and Morrison, P. J. [2000]. Classification and Casimir invariants of Lie-Poisson brackets. Physica D 136, 205-244.

Trinãnes, J., Putman, N. F., Goni, G. J., Hu, C. and Wang, M. [2021]. Monitoring pelagic Sargassum inundation potential for coastal communities. Journal of Operational Oceanography doi:10.1080/1755876X.2021.1902682, 1-12.

Wang, M. and Hu, C. [2016]. Mapping and quantifying Sargassum distribution and coverage in the Central West Atlantic using MODIS observations. Remote Sens. Environ. 183, 350-367.

Wang, M., Hu, C., Barnes, B., Mitchum, G., Lapointe, B. and Montoya, J. P. [2019]. The Great Atlantic Sargassum Belt. Science 365, 83-87. 